\title{
Texture development mechanisms in ion beam assisted deposition
}

\author{
L. Dong and D. J. Srolovitz ${ }^{\text {a) }}$ \\ Department of Materials Science and Engineering, The University of Michigan, Ann Arbor, \\ Michigan 48109-2136
}

(Received 19 May 1998; accepted for publication 10 July 1998)

\begin{abstract}
Three-dimensional molecular dynamics simulations of ion beam assisted deposition (IBAD) are performed to determine the mechanisms of crystallographic texture selection during the IBAD of polycrystalline films. A face centered cubic bicrystal consisting of [111] and [110] oriented grains is grown while an ion beam bombards the growing film at normal incidence. As the film grows, the grain boundaries delimiting the [111] and [110] grains move towards each other, eventually pinching off the [111] grain such that the film texture changes from equal densities of [111] and [110] to purely [110]. Examination of single crystals grown in the presence of ion beams shows two important effects: ion beam induced atomic sputtering from the surface and ion beam induced damage are significantly reduced when the ion beam is oriented along channeling directions of the crystals. The first observation suggests that grains with channeling directions aligned parallel to the ion beam grow more quickly than those where they are not aligned. This leads to grain-to-grain variations in the film thickness that increase in magnitude during growth. Variations in thickness result in a shadowing effect that further slows the growth of the less thick (nonaligned) grainseventually leading to pinch-off of these grains. The second observation suggests that the stored energies within the grains with channeling directions aligned parallel to the ion beam will be lower than that of the nonaligned grains. This difference in stored energy (in the form of crystal defects) is shown to lead to grain boundary migration - a process equivalent to primary recrystallization. Both of these effects can lead to changes in crystallographic texture during film growth and both are observed in the bicrystal simulations. Which mechanism will dominate under a prescribed set of conditions remains to be delineated. (C) 1998 American Institute of Physics.
\end{abstract}

[S0021-8979(98)01020-2]

\section{INTRODUCTION}

Polycrystalline thin films, grown by almost any method, exhibit at least some degree of crystallographic texture. Most commonly, the observed texture corresponds to a fiber texture, in which the surface normals of the grains are (nearly) parallel to a specific crystallographic direction (i.e., out-ofplane texture) and the in-plane orientations of the grains are nearly random. The crystallographic orientation of the outof-plane texture of a given material can vary with film growth method. Ion beams have been employed to control the out-of-plane texture. ${ }^{1-8}$ In the present article, we examine the effects of ion beam energy, orientation, and flux on the development of out-of-plane texture and use these results to interpret the underlying mechanisms for texture development during the ion beam assisted deposition (IBAD) of polycrystalline films.

Fiber textures are commonly observed in polycrystalline thin films grown by physical vapor deposition (PVD) techniques. ${ }^{9-13}$ For example, face centered cubic (fcc) metal films often exhibit a $\langle 111\rangle$ preferred orientation, while metallic body centered cubic (bcc) films commonly develop a $\langle 110\rangle$ preferred orientation. The existence of these fiber textures is usually explained in terms of surface energy arguments. $\{111\}$ surfaces in fcc materials and $\{110\}$ surfaces in

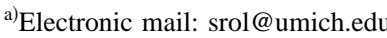

bcc materials correspond to the most densely packed planes in each lattice and, hence, to the lowest energy surfaces. Therefore, it is thermodynamically favorable to orient all of the grains in the film such that their surface normal is perpendicular to these highest density planes. ${ }^{13}$ This idea was supported by recent simulations ${ }^{14}$ which showed a net surface diffusion flux of atoms toward grains with low energy surfaces at the expense of their neighbors, leading to the preferential growth of these grains. The variation of the binding energy of surface atoms with surface orientation can also lead to differences in sputtering rates when relatively high energy species are present in the growth chamber. ${ }^{15}$ This too can result in variations of growth rate with surface orientation. This effect, coupled with shadowing, ${ }^{16}$ can also affect the film texture. ${ }^{17}$

Ion beams can be used to modify the texture that normally develops during physical vapor deposition. ${ }^{1-8} \mathrm{Yu}$ et $a l^{3,4}$ used low angle ion bombardment during growth to modify film texture. They argued that the texture was controlled by the difference in sputtering yield between grains of different orientations (with respect to the ion beam). This difference leads to higher growth rates for grains that sputter less than the average. Bradley et al. ${ }^{18,19}$ developed a model for texture development based upon this variation of sputtering yield with grain orientation. In their model, the variation of sputtering yield with grain orientation is associated with the existence of channeling directions, i.e., crystallographic 
directions along which ions can penetrate relatively deeply into the crystal. For example, an ion beam oriented normal to an fcc film will cause a shift in orientation from a $\langle 111\rangle$ (i.e., that favored by surface energy considerations) to a $\langle 110\rangle$ texture, since the easiest channeling direction is $\langle 110\rangle$ for simple fcc crystals. Experiments on many materials, including diamond ${ }^{20}$ and copper ${ }^{21}$ single crystals, have shown that the sputtering yield is indeed a strong function of the angle of incidence of the ion beam for ion beam energies as low as several hundred $\mathrm{eV}$ through more than $20 \mathrm{keV}{ }^{22}$ The sputtering yield commonly decreases by between two and five times when the ion beam direction is parallel to a channeling direction of a crystal.

Van Wyk and Smith ${ }^{1}$ studied the development of preferred orientation in $\mathrm{Cu}$ films which were vacuum deposited and then bombarded with $40 \mathrm{keV} \mathrm{Cu}^{+}$ions. Upon ion bombardment, these films changed from a strong $\langle 111\rangle$ texture to a $\langle 220\rangle$ texture. They suggested that the $\langle 110\rangle$ oriented grains were damaged less by the ions than grains with other orientations because $\langle 110\rangle$ is the easiest channeling direction in $\mathrm{Cu}$. During the thermal spike associated with the ion bombardment, the relatively perfect $\langle 110\rangle$ grains grow into their more damaged surroundings by a recrystallization process, thereby reorienting the more damaged material to $\langle 110\rangle$. Dobrev $^{2}$ drew the same conclusions based on his observations of texture changes during $10 \mathrm{keV} \mathrm{Ar}^{+}$bombardment of vapor deposited fcc and hep metal films. While Yu et al. ${ }^{3,4}$ and Bradley et al. ${ }^{18,19}$ focused on the effects of low energy ion bombardment during growth, Van Wyk and Smith ${ }^{1}$ and Dobrev $^{2}$ experiments investigated the effects of postgrowth, high energy ion bombardment. Taken in toto, these studies suggest that the crystal orientation dependence of ion beam effects is associated with channeling, although the mechanism(s) by which the ion beam modifies texture remains uncertain.

Several atomic-scale computer simulations of ion beam assisted deposition have been performed (see, e.g., Refs. 2332 ). Both molecular dynamics (MD) ${ }^{24-28}$ and binary collision approximation $(\mathrm{BCA})^{29-32}$ simulations have been performed to investigate the effects of ion bombardment on film densification, radiation damage, sputtering, ion mixing, and implantation. In the present study, we extend these earlier atomistic simulation studies to examine the effects of ion beams on texture evolution. We perform a series of threedimensional molecular dynamics simulations on both bicrystals and single crystals designed to clarify the mechanisms for ion beam texture control and to identify the ion beam parameters for which these mechanisms operate.

\section{METHOD}

A three dimensional (MD) simulation program for ion beam assisted deposition has been developed based on an MD simulation program described in earlier publications. ${ }^{17,33}$ Only a brief description is presented here. The MD simulations were performed by integrating Newton's classical equation of motion for each atom forward in time using a fifth order Nordsiek, predictor-corrector method. Since, in this study, we focused on the generic features of ion beam assisted film growth, rather than the detailed nature of any particularly material, we employed simplistic, but well understood descriptions of the atomic interactions. The potential energy describing the interaction between atoms was the classical Lennard-Jones pair potential,

$$
U\left(r_{i j}\right)=\epsilon\left[\left(\frac{r_{0}}{r_{i j}}\right)^{12}-2\left(\frac{r_{0}}{r_{i j}}\right)^{6}\right],
$$

where $U\left(r_{i j}\right)$ is the interaction energy between atoms $i$ and $j$ separated by distance $r_{i j}, \epsilon$ scales the strength of the interaction, and $r_{0}$ is the characteristic length of the potential. This potential was smoothly cut off at $r=2.1 r_{0}$. The present simulations were performed using parameters appropriate for $\mathrm{Al}$ atoms and $\mathrm{Ne}^{+}$ions. The interactions between the atoms and ions and between the ions were described using the purely repulsive Molière potential ${ }^{34}$

$$
\begin{aligned}
V\left(r_{i j}\right)= & \frac{Z_{1} Z_{2} e^{2}}{r_{i j}}\left[0.35 e^{-0.3 r_{i j} / a}+0.55 e^{-1.2 r_{i j} / a}\right. \\
& \left.+0.1 e^{-6.0 r_{i j} / a}\right],
\end{aligned}
$$

where $a=0.468\left(Z_{1}^{1 / 2}+Z_{2}^{1 / 2}\right)^{-2 / 3}$ is the Firsov screening length, and $Z_{1}$ and $Z_{2}$ are the atomic numbers of the ions and film atoms, respectively. We chose the atomic numbers $Z_{1}$ and $Z_{2}$ to represent $\mathrm{Ne}$ and $\mathrm{Al}$, respectively. The $\mathrm{Ne}-\mathrm{Al}$ and $\mathrm{Ne}-\mathrm{Ne}$ interactions were truncated at $r=2.1 r_{0}$, respectively.

The basic parameters describing the atomic interactions in this simulation are the atomic mass $M$, the atomic potential well depth $\epsilon$, and the characteristic length $r_{0}$. The velocity of the deposited atoms (ions) is $V_{\text {atoms }}=\sqrt{2 E_{a} / M_{a}}$ $\left(V_{\text {ions }}=\sqrt{2 E_{i} / M_{i}}\right)$ and the basic unit of time is $\tau$ $=\sqrt{M_{a} r_{0}^{2} / \epsilon}$, where $E_{a}\left(E_{i}\right)$ is the energy of the beam of atoms (ions). $E_{a}$ was fixed at $E_{a}=1 \epsilon /$ atom throughout this study. The time step was variable and was determined such that the maximum particle displacement at each time step was $\Delta r_{\max }=r_{0} / 200=V_{\max } \Delta t$, where $V_{\max }$ is the velocity of the fastest particle at each time step. In order to give a physical feel for these parameters in terms of a real material, we have estimated these values for $\mathrm{Al}$ as follows: $r_{0}=0.286 \mathrm{~nm}, \epsilon=0.565 \mathrm{eV}, M_{a}=4.48 \times 10^{-26} \mathrm{~kg}$, and $\tau$ $=2.0 \times 10^{-13} \mathrm{~s}$. All of the results reported below are in terms of the fundamental parameters $r_{0}, \epsilon$, and $M_{a}$.

The computational cell was three dimensional with the $z$ direction perpendicular to the (flat) initial crystal surface. Periodic boundary conditions were employed along $x$ and $y$ directions and open (or free) boundary conditions were employed in the $+z$ direction. The incident ion beam was directed onto the growing crystal at predetermined angles (see Fig. 1) and the atomic deposition flux was oriented in the $x-z$ plane at $45^{\circ}$ to the $z$ axis. The atoms in the initial crystal (Al) were arranged on an fcc lattice. The initial crystal consists of a fixed number of atomic layers. The atoms in the bottom few layers of the initial crystal were frozen in space to prevent the crystal from translating through space due to the momentum absorbed from the deposition flux. Atoms in the next several layers were "thermostated" in order to maintain the system at the desired temperature. ${ }^{17,33}$ As the film grows, the thickness of the thermostated region was increased to prevent excessive heating of the film due to the kinetic en- 


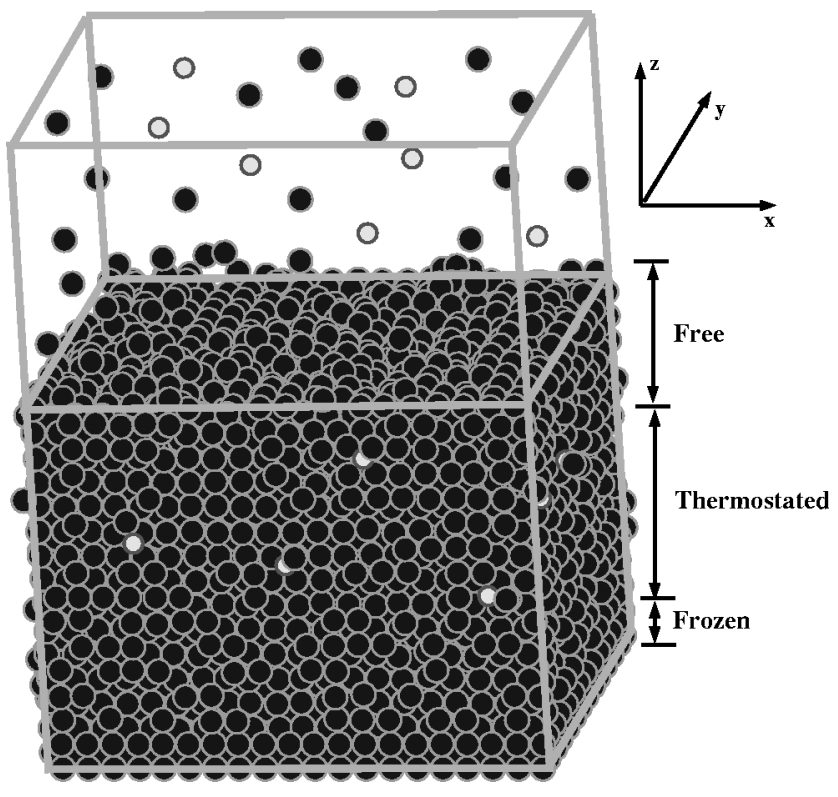

FIG. 1. An illustration of the computational cell, depicting the initial crystal, growing film, atoms impinging onto the surface, and bombarding ions. The larger/darker particles are atoms and the smaller/gray ones are ions. The bottom few atomic layers of the initial crystal are frozen, the layers above these are thermostated and the atoms in several layers near the free surface are unconstrained. The thickness of the thermostated region increases as the film grows.

ergy and bonding energy associated with incorporation of vapor atom into the solid. The surface region was not thermostated in order to insure that the dynamical features of the film growth process were not biased by the artificial dynamics inherent in all thermostating procedures. While this may lead to a temperature gradient, the large mass of the thermostated region and the small thickness of the unthermostated region $\left(15 r_{0}\right)$ ensures that such temperature gradients are small.

The deposition flux and the ion beam were turned on and off alternatively in order to fix the ion-to-atom arrival ratio $R$ (i.e., the ratio of the number of deposited ions to the number of deposited atoms). Since the ions possessed relatively large kinetic energies, they frequently reflected off the surface or ejected other surface atoms back up into the vapor. All such atoms and ions were removed from the vapor.

Since the MD time scale is, by necessity, very short, we employed large deposition rates in the simulations. We prevented gas phase reactions from occurring by (1) collimating the deposition beam, (2) switching on the ion beam only when all deposition atoms have reached the film surface, and (3) by removing atoms that were either ejected or reflected from the surface. While high deposition rates do not allow sufficient time for realistic atomic diffusion at typical deposition temperatures, this was overcome, in part, by performing the simulations at somewhat elevated temperatures: note, there is an exponential increase in the surface diffusion length with increasing temperature and only an inverse square root variation with deposition rate.

\section{BICRYSTAL SIMULATIONS}

The goal of the present study is to use atomistic simulations to understand the mechanisms by which the ion beam influences the film texture during ion beam assisted deposition. We begin by demonstrating that the presence of an ion beam does indeed modify the film orientation during deposition. To this end, we simulated the growth of a bicrystal film. The bicrystal was $24.8 d_{0}$ along the $x$ direction, $15.59 d_{0}$ in the $y$ direction, and $6.5 d_{0}$ in the $z$ direction and the grain boundaries lie along the $y-z$ plane at $x=0$ and $x$ $=12.4 d_{0}$, where $d_{0}$ is the equilibrium lattice parameter at the thermostated temperature $\left(0.4 \epsilon / k_{B}\right.$, where $k_{B}$ is Boltzmann's constant and the zero pressure melting temperature is $\left.\sim 0.7 \epsilon / k_{B}\right)$. One crystal is oriented with the [111] direction parallel to the $z$ axis and the $[\overline{1} 10]$ and $[\overline{1} 12]$ directions along the $x$ and $y$ axes, respectively. The other crystal is oriented with the [110] direction parallel to the $z$ axis and the $[\overline{1} 10]$ and [001] directions along the $x$ and $y$ axes, respectively. The films were grown with $E_{i}=800 \epsilon /$ ion, $R=1 / 2$ and an average atom deposition rate of 1.25 atom $/ \tau$. The ion beam was oriented perpendicular to the nominal surface (i.e., the $-z$ direction). This direction is parallel to the [110] channeling direction in the [110] oriented grain (i.e., the easiest channeling direction for the fcc crystal) and the nonchanneling [111] direction in the [111] oriented grain. ${ }^{35}$

The temporal evolution of the atomic structure of the bicrystal is shown in Fig. 2. Figure 2(a) shows the bicrystal before deposition: the [110] and [111] grains are on the left and right sides, respectively, and are separated by relaxed grain boundaries (one on the far left, due to the periodic boundary conditions). Following deposition and ion bombardment, some ions (the small, light gray particles are the ions) are embedded within the crystals and significant damage to the crystals is evident [see Figs. 2(b)-2(d)]. Clearly the [111] grain suffers much more ion-induced damage than the [110] grain [Fig. 2(b)]. While the atoms in the top layers of the [111] grain are significantly displaced from their equilibrium lattice positions, most of the atoms near the [110] grain surface remain on their lattice sites. Examination of Fig. 2(c) shows that the crystal structure of the [111] grain has recovered somewhat from the damage seen in Fig. 2(b) and that both grain boundaries have tilted toward the center of the [111] grain. As a result, the [111] grain is much smaller at the top than it was originally, thereby demonstrating that the [110] grain grows at the expense of the [111] grain. It is also evident in Fig. 2(c) that the [111] grain is not as thick (high) as the [110] grain. Figure 2(d) shows that at late times, the two grain boundaries are touching (or nearly touching) such that that the [111] grain is effectively occluded by the [110] grain. This series of images clearly demonstrates that $\langle 110\rangle$ oriented grains in fcc solids will grow at the expense of other grains that are not oriented in favorable channeling directions during IBAD with the ion beam directed normal to the nominal surface. This will lead to the formation of a $\langle 110\rangle$ fiber texture.

Based upon the atomic structures shown in Fig. 2, it is obvious that channeling plays a key role in determining the texture of films grown by IBAD, at least in the energy range of the present simulations. As mentioned briefly above, the role which channeling plays in texturing may be associated with (i) sputtering induced differences in growth rate rect,18,19 $^{3,4}$ and subsequent shadowing ${ }^{17}$ or (ii) the variation of ion dam- 


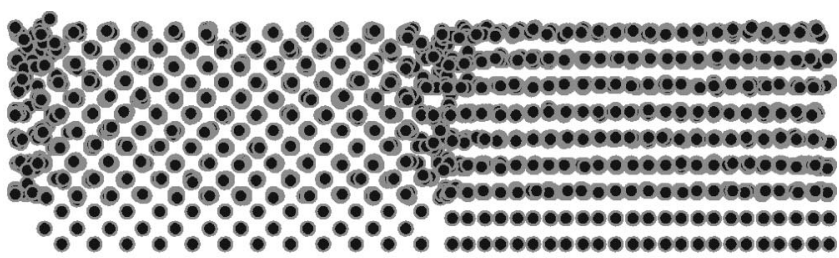

(a)

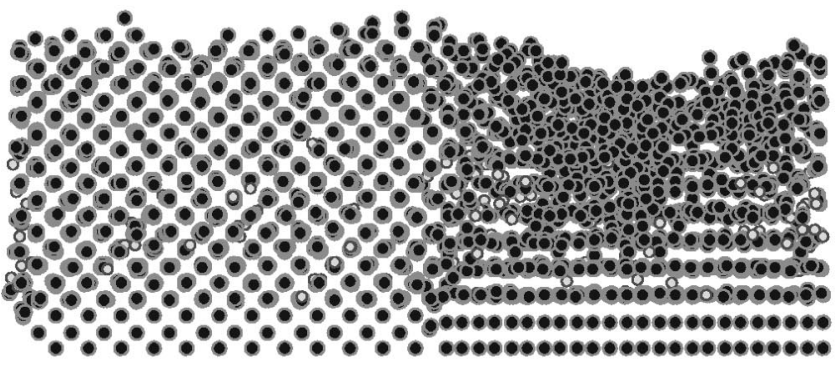

(b)

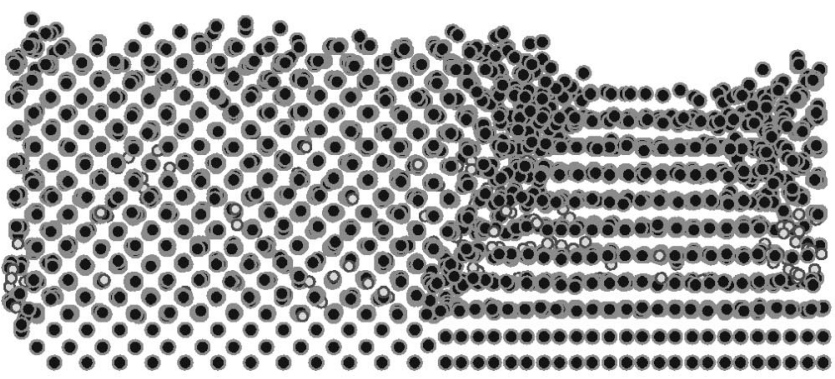

(c)

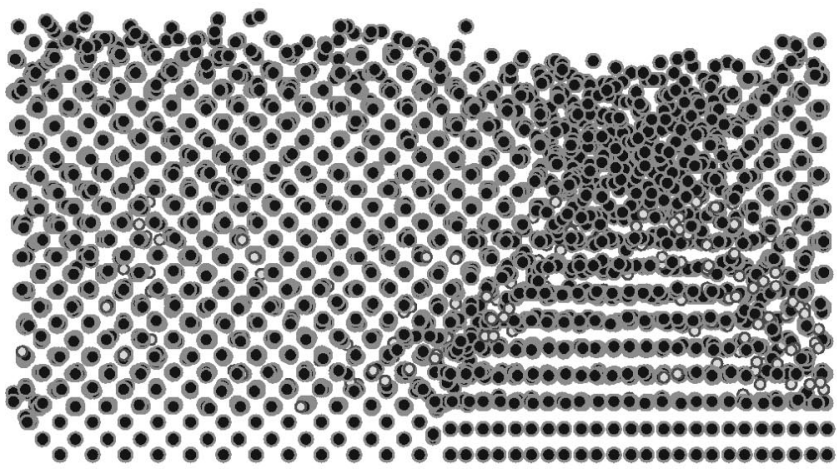

(d)

FIG. 2. (a) The atomic structure of the bicrystal viewed along the $y$ direction at $T=0.4 \epsilon / k_{B}$. The atomic size is reduced to show the inner structure of the crystal. The crystal on the left is oriented such that the [110] direction is normal to the free surface and the crystal on the right has a [111] surface normal. Because periodic boundary conditions are employed in the $x$ and $y$ directions, there are grain boundaries (parallel to the $y-z$ plane) in the center of the figure and on the edges (left and right edges are equivalent). The kinetic energy of the deposition flux $E_{a}=1 \epsilon /$ atom and the atoms are deposited at an angle of $\alpha=45^{\circ}$ with respect to the nominal surface normal in the $x-z$ plane. The ion beam is oriented normal to the bicrystal surface, such that it is aligned with the [110] channeling direction of the [110] oriented grain and no channeling directions of the [111] oriented grain. The ion-to-atom arrival ratio $R=1 / 2$ and the energy of the ion beam $E_{i}=800 \epsilon /$ ion. (b) Structure of the bicrystal during IBAD at $t=1229 \tau$. (c) Structure of the bicrystal during IBAD at $t=1471 \tau$. (d) Structure of the bicrystal during IBAD at $t$ $=2148 \tau$.

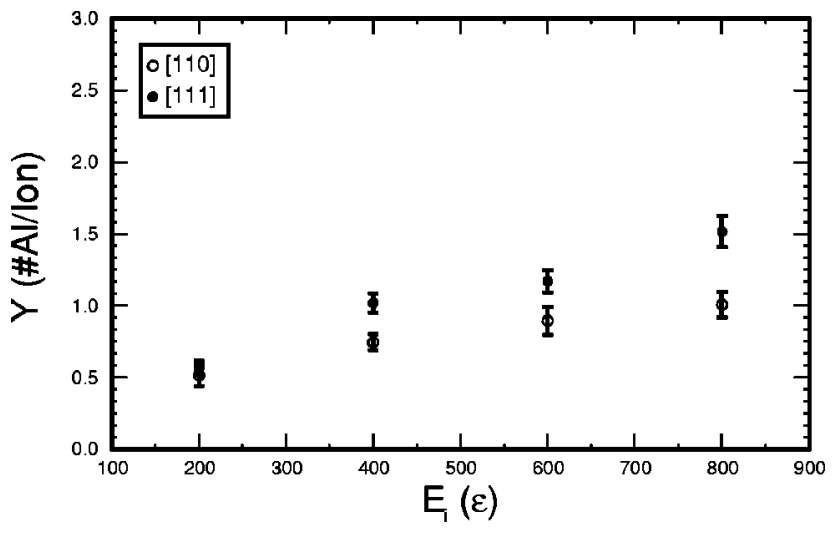

FIG. 3. Sputtering yield from [110] (open symbols) and [111] (filled symbols) oriented single crystals with a normal incidence ion beam and $R$ $=1 / 5$. The ion beam is parallel to the [110] channeling direction of the [110] crystals and is not parallel to any channeling directions of the [111] crystals. The error bars represent plus and minus one standard deviation of the data from three independent simulations.

age with crystal orientation leading to recrystallization-like grain boundary migration. The present results show that there is indeed a difference in growth rate between differently oriented grains [see Fig. 2(c)], thereby supporting mechanism (i). At the same time, Figs. 2(b)-2(d) clearly show pronounced differences in the amount of damage with grain orientation and grain boundary migration toward the more damaged grain, thereby supporting mechanism (ii). In order to distinguish between the two mechanisms, we performed a series of simulations to investigate the mechanisms separately.

\section{SPUTTERING INDUCED THICKNESS VARIATION}

In order to quantify the effect of crystal orientation on the sputtering yield (consistent with the bicrystal simulations presented above), we performed a series of IBAD simulations on [111] and [110] oriented single crystals. The [111] oriented crystal dimensions were $15 d_{0} \times 15.59 d_{0} \times 9.8 d_{0}$ in the $x$ [110], $y$ [112], and $z$ [111] directions, respectively. The [110] oriented crystal was $15 d_{0} \times 15.56 d_{0} \times 10 d_{0}$ in the $x$ [110], $y$ [001], and $z$ [110] directions, respectively. We employed the following parameters during these simulations: $T=0.4 \epsilon / k_{B}, E_{a}=1 \epsilon /$ atom, and the atomic flux was oriented $45^{\circ}$ from the $z$ axis within the $x-z$ plane.

We performed a series of simulations with different ion beam orientations relative to the growing film. We first examined the case of a normal incidence ion beam (as per our bicrystal simulations), corresponding to the easy channeling direction for the [110] crystal and a nonchanneling orientation for the [111] crystal. In these simulations, the ion-toatom arrival ratio was fixed at $R=1 / 5$. The sputtering yield $Y$ (the ratio of the number of sputtered atoms to the number of ions) versus ion energy $E_{i}$ is shown in Fig. 3. The sputtering yield increases with increasing ion energy for both [111] and [110] oriented films. The [111] sputtering yield is greater than the [110] sputtering yield. The difference between the [111] and [110] sputtering yield increases with increasing ion energy. This is a clear indication that $\langle 111\rangle$ oriented films should grow more slowly than $\langle 110\rangle$ oriented films during 


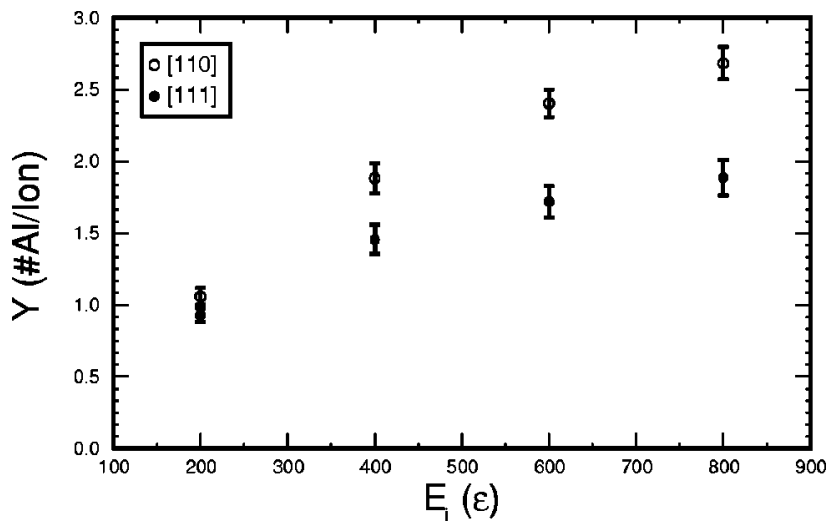

FIG. 4. Sputtering yield from [110] (open symbols) and [111] (filled symbols) oriented single crystals with the ion beam oriented at $35.26^{\circ}$ with respect to the surface normal within the $y-z$ plane and $R=1 / 5$. The ion beam is parallel to the [110] channeling direction of the [111] crystals and is not parallel to any channeling directions of the [110] crystals.

ion beam assisted deposition when the ion beam is oriented normal to the film surface. During polycrystalline film growth, this will lead to grain-to-grain variations in thickness (with $\langle 110\rangle$ oriented grains thicker than $\langle 111\rangle$ oriented grains).

We performed two additional sets of simulation to confirm the effects of relative crystal and ion beam orientation on sputtering yield. In the first case, we oriented the ion beam at $35.26^{\circ}$ to the $z$ axis within the $y-z$ plane, which correspond to a $\langle 110\rangle$ channeling direction in the [111] oriented film and a nonchanneling direction in the [110] oriented film. Figure 4 shows that with this ion beam orientation there is more sputtering from the [110] film as compared with the [111] film and that this difference increases with increasing energy. This result demonstrates that any crystal orientation can be favored by the ion beam, depending on the choice of ion beam orientation. Finally, Fig. 5 shows the [111] and [110] sputtering yield versus ion energy for the case of an ion beam oriented in nonchanneling directions for both [111] and [110] films (i.e., at $30^{\circ}$ to the $z$ axis in the $x-z$ plane). In this case, there is essentially no difference in the

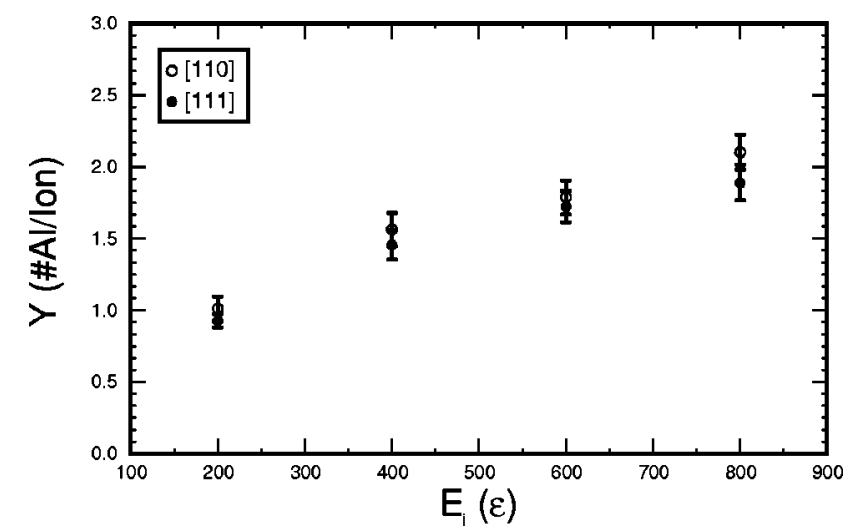

FIG. 5. Sputtering yield from [110] (open symbols) and [111] (filled symbols) oriented single crystals with the ion beam oriented at $30^{\circ}$ with respect to the surface normal within the $x-z$ plane and $R=1 / 5$. The ion beam is not parallel to any channeling direction in either the [111] or [110] oriented crystals.

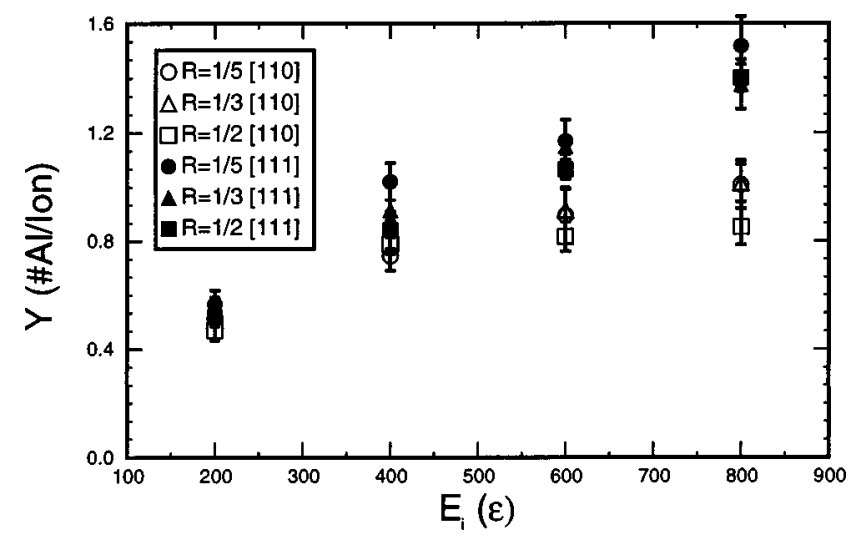

FIG. 6. The sputtering yield from [110] (open symbols) and [111] (filled symbols) oriented single crystals with the ion beam oriented normal to the surface for $R=1 / 2$ (squares), 1/3 (triangles), and 1/5 (circles).

sputtering yield for the two film orientations, as expected based upon our channeling argument for sputtering yield.

All of the data shown above was obtained for the case where the ion-to-atom arrival ratio $R=1 / 5$. Figure 6 shows that variations in $R$ do not significantly effect the sputtering yield. This removes one experimental variable from consideration. We also examined whether the number of experimental variables could be reduced even further by plotting the fraction of deposited atoms that are sputtered from the surface $Y R$ as a function of the composite parameter $E_{i} R$, the total ion energy per deposited atom (Fig. 7), as is commonly done experimentally. This reduction in the number of variables appears valid for $\langle 111\rangle$ oriented crystals, but not for $\langle 110\rangle$ oriented crystals, with a normal incidence ion beam. The origin of this effect may be found in the shape of the $Y$ vs $E_{i}$ plot (Fig. 6). $Y$ is nearly a linear function of $E_{i}$ for $\langle 111\rangle$ oriented films and $Y$ is a substantially sublinear function of $E_{i}$ for $\langle 110\rangle$. YR vs $E_{i} R$ will only be independent of $R$, if $Y$ is a linear function of $E_{i}$ for all $R$. Therefore, plotting the data in terms of the reduced variable, total ion energy deposited per atom $E_{i} R$, is in general, inappropriate. The origin of the difference in the $E_{i}$ vs $R$ (and, hence, $Y R$ vs $E_{i} R$ ) behavior of the $\langle 111\rangle$ and $\langle 110\rangle$ oriented (with normal

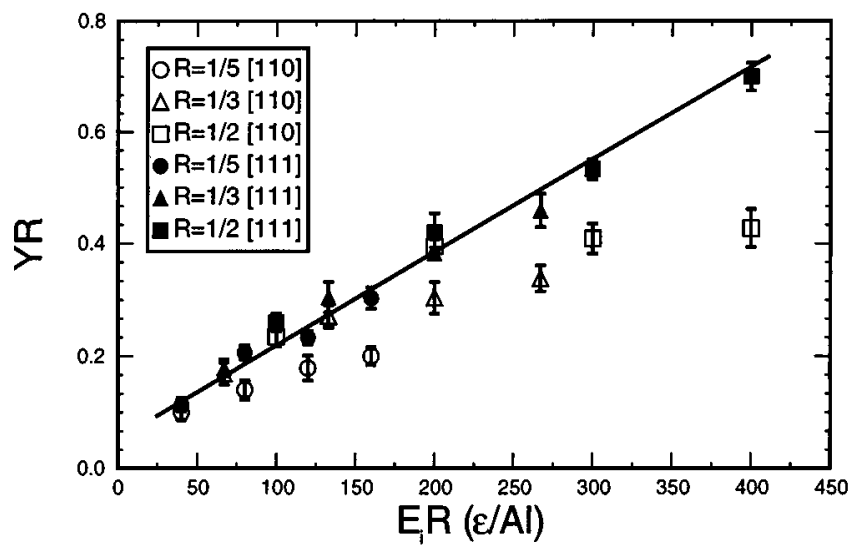

FIG. 7. The fraction of deposited atoms that are sputtered from the surface, $Y R$, as a function of the total ion energy per deposited atom, $E_{i} R$. The filled symbols correspond to [110] oriented crystals and the open symbols correspond to [111] oriented crystals. The ion beam is oriented normal to the surface and $R=1 / 2$ (squares), 1/3 (triangles), and 1/5 (circles). 
incidence ions) is likely associated with the difference in the effects of ion beams in channeling and nonchanneling directions.

The sputtering yield difference between the grains aligned such that their channeling directions are parallel to the ion beam and those that are not aligned translates into thickness differences between the grains that increase during growth. Therefore, $\langle 111\rangle$ oriented grains will be less thick than $\langle 110\rangle$ grains when the ion beam is oriented normal to the nominal surface. This is consistent with our bicrystal observations [see Fig. 2(c)]. When the thicknesses of neighboring grains differ, shadowing effects become important. While this is true when the deposition flux is normal to the nominal surface, ${ }^{17}$ it becomes very pronounced for oblique deposition (as in Fig. 2) and when the deposition flux has a wide angular spread. ${ }^{16}$ Shadowing effectively decreases the deposition atom flux to the less thick grains, thereby increasing the difference in growth rate between the thick (ion beam aligned to channeling direction) and thin (nonaligned) grains. This effect feeds back on itself since shadowing increases growth rate difference which, in turn, leads to more shadowing. This process will eventually lead to the aligned grains overgrowing the nonaligned ones. For ion beams that are normal to the nominal surface, this leads to $\langle 110\rangle$ grains dominating the texture at the expense of $\langle 111\rangle$ grains. This is consistent with the argument put forward by Ying et al. ${ }^{17}$ based on two dimensional MD simulations with an energetic deposition flux (rather than with energetic ions, as in the present case).

\section{RECRYSTALLIZATION}

The lattice images in the bicrystal simulations (Fig. 2) show that the $\langle 111\rangle$ oriented crystals were much more damaged by the ion beam than were the $\langle 110\rangle$ oriented crystals. To quantify this observation, we performed a series of simulations in which we subjected [111] and [110] single crystals to an ion beam and then characterized the resulting damage. The dimensions of the crystals are the same as those used in studying sputtering yield, above. In these simulations, we equilibrated the crystals at $T=0.4 \epsilon / k_{B}$, bombarded them with 20, normal incidence, ions (with no deposition flux) and then recorded the temporal evolution of the structure. Each ion initially had a kinetic energy of $1000 \epsilon$. Figure 8 shows the atomic structure of the [110] and [111] oriented crystals at $t=1 \tau$ after the ion impact. Clearly the [111] oriented crystal is damaged much more than the [110] oriented one. To quantify the degree of disorder wrought by the ions, we measured the radial distribution function $G(r)$ within $5.1 d_{0}$ of the surfaces (where the damage is the greatest). We employed the following definition of the radial distribution function:

$$
G(r)=4 \pi r\left[\rho(r)-\rho_{0}\right],
$$

where $\rho_{0}$ is the average density and $\rho(r)$ is calculated by successively fixing the origin on each atom within the system and calculating the density of atoms at a distance $r$ from this origin. Figure 9 shows $G(r)$ for the [110] and [111] oriented crystals corresponding to the structures shown in Fig. 8. The

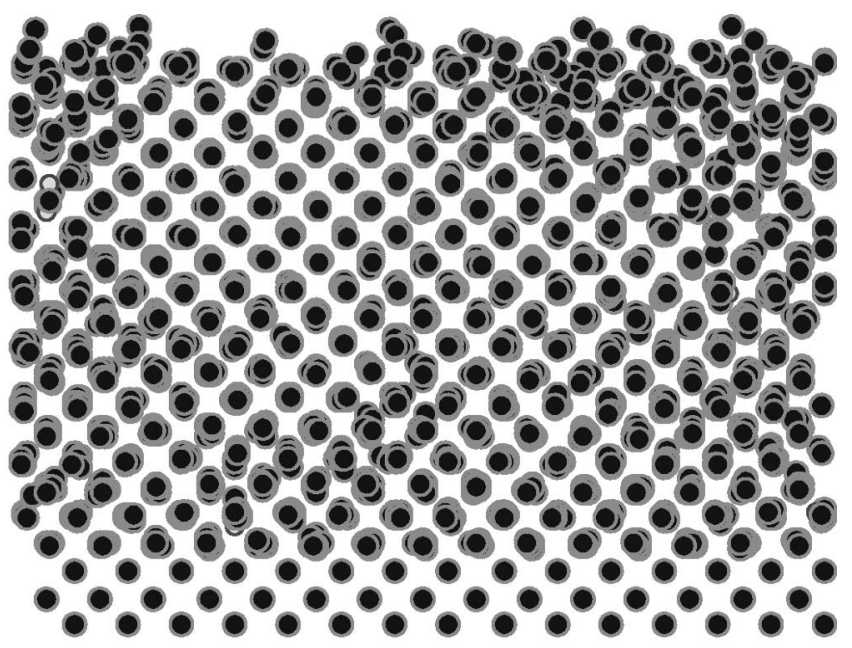

(a)

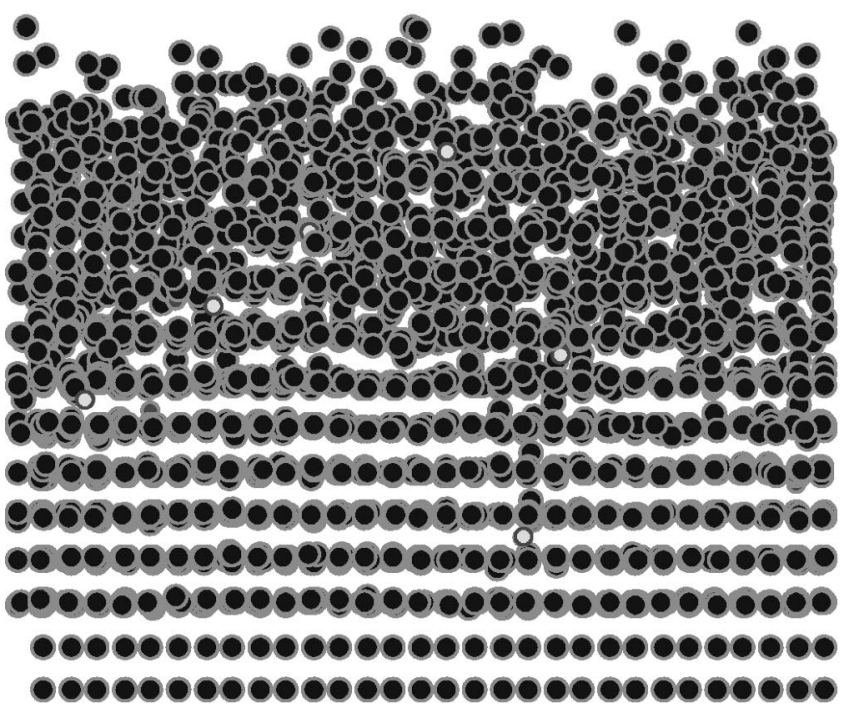

(b)

FIG. 8. The atomic structure of the (a) [110] and (b) [111] oriented crystals, $1 \tau$ after a pulse of 20 ions impacted the surface. The ion beam is oriented normal to the crystal surfaces: i.e., aligned with the [110] channeling direction of the [110] single crystals and not aligned with any channeling direction of the [111] single crystals. The ion energy is $1000 \mathrm{\epsilon} / \mathrm{ion}$.

magnitudes of the peaks and valleys of $G(r)$ for the [110] oriented crystal are much greater than those for the [111] crystal. This indicates that the atoms in the [111] crystal are displaced from their equilibrium positions much more than those in the [110] crystal. The damage created by the ions evolves with time, as shown in Fig. 10, where we plot the height of the first peak in $G(r), P_{1}(t)$, versus time (normalized by the peak height of the perfect crystal at $\left.T=0.4 \epsilon / k_{B}\right)$. The peak height drops rapidly at very early times, while the kinetic energy of the ions are converted into heat and damage, and then slowly recovers over a much larger time scale. While most of the damage heals relatively quickly, some residual damage or defects remain at long times. The magnitude of the initial damage is greater for the [111] grains than for the [110] grains. At long times, the damage that remains within the [111] crystals is larger than that in the 


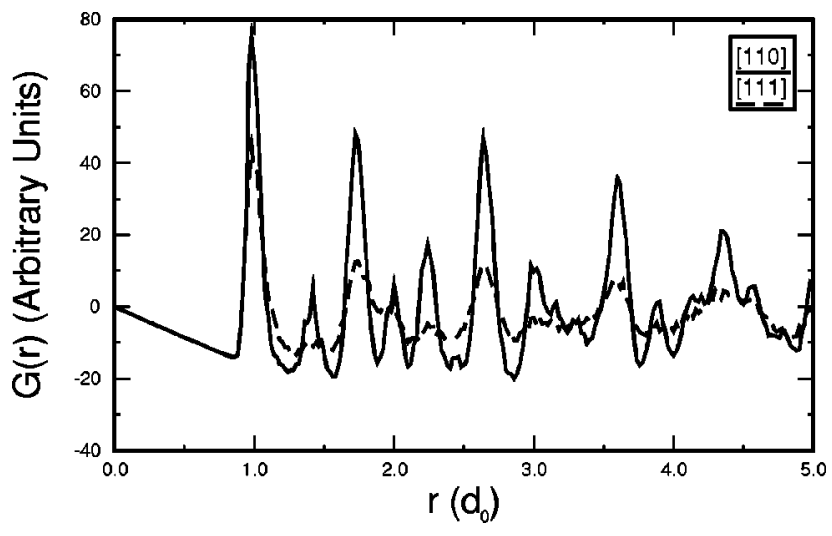

FIG. 9. Radial distribution functions $G(r)$ for the single crystals damaged by the ion beam pulse shown in Fig. 8. The radial distribution function was only averaged over atoms within $5.1 d_{0}$ of the surface.

[110] crystals. Given sufficient time, all of the damage should eventually anneal out. This time is much longer than is accessible by the present, very short MD simulations and, hence, a difference in defect density persists to the end of the simulations. In IBAD experiments, the time scales are also not large enough to anneal out all of the ion beam induced damage during the deposition. Therefore, the $\langle 111\rangle$ crystals will exhibit a larger steady state defect density than will the $\langle 110\rangle$ crystals.

In addition to determining the radial distribution functions, we also measured the ion beam induced damage in terms of changes in the energy of the crystal. We prepared and ion bombarded the [110] and [111] crystals in the same manner as described in the preceding paragraph and measured the average energy per atom in the $5.1 d_{0}$ layer adjacent to the surface as a function of time. Since we are interested in the energy of the defected crystals and not the thermal energy, we quenched the samples to $T=0 \mathrm{~K}$ prior to each energy measurement. The resultant energy per atom $E_{a}$ is plotted in Fig. 11 as a function of time following the ion bombardment. Prior to the ion bombardment, the energy of this layer is $-6.371 \epsilon$ for the $[110]$ crystal and the $-6.423 \epsilon$ for the [111] crystal (the difference is due to the variation of surface energy with orientation). These data show that the

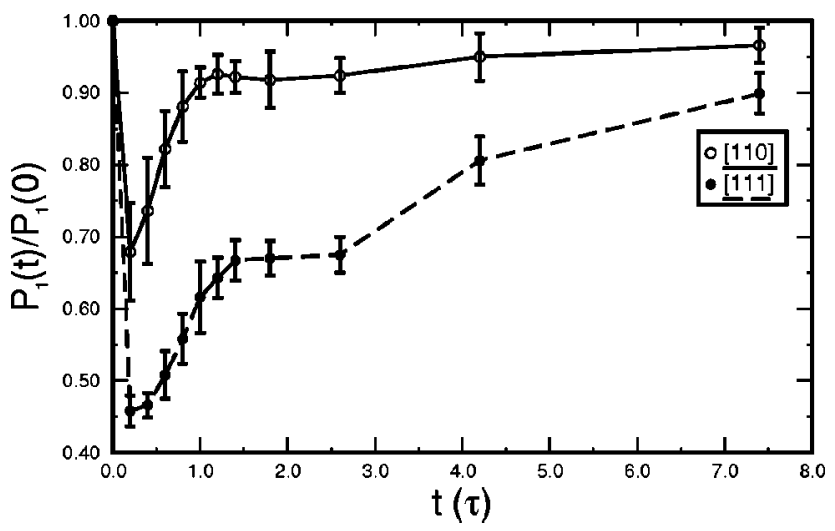

FIG. 10. The height of the first peak of the radial distribution function, $P_{1}(t)$, vs time following the ion beam pulse (normalized by the peak height of the perfect crystal at $T=0.4 \epsilon / k_{B}$ ).

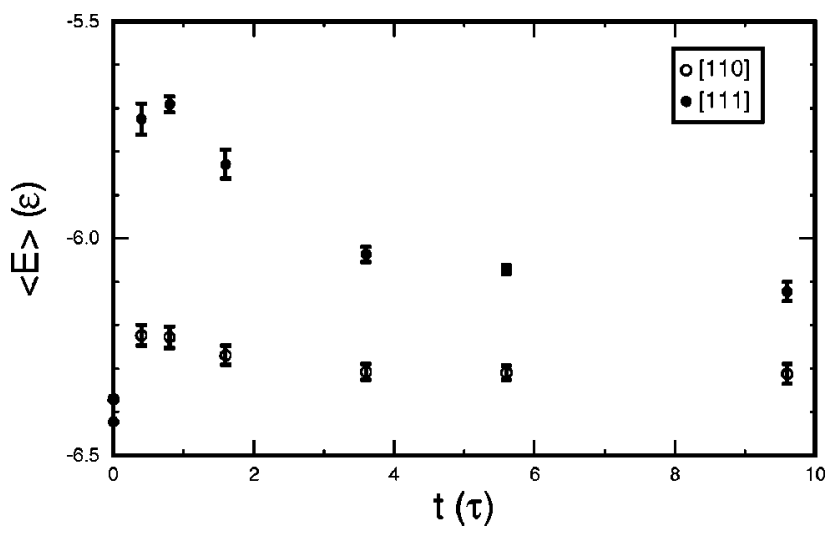

FIG. 11. The average energy per atom $\langle E\rangle$ within $5.1 d_{0}$ of the surface of single crystals following the ion beam pulse (see Fig. 8). The average energies were evaluated after the crystals were quenched to $T=0$.

energies of the crystals rapidly increase with time following the ion impact and then slowly decays to an energy which is higher than the initial crystal energy. This general form is consistent with that observed for the radial distribution peak heights (see Fig. 10). Figure 11 demonstrates that the ion beam inflicts significantly more damage on the [111] crystal (the channeling directions of which are not aligned with the ion beam) than on the [110] crystal, where the damage is minimal (aligned with respect to the ion beam). The total amount of damage to the [110] crystal is minimal, thereby indicating that the initial large drop in $P_{1}(t)$ for the [110] crystal is associated with large amplitude atomic vibrations and not significant defect generation. The differences between the energies of the [110] and [111] crystals persist to the longest times accessed in the simulations. As discussed with respect to the radial distribution function, these energy differences are expected to lead to steady state energy differences between the grains with channeling directions aligned with the ion beam and those which are not in constant ion beam IBAD experiments.

The significance of a difference in stored energies in crystals of different orientation is associated with the role it plays as a driving force for recrystallization. In primary recrystallization, grain boundaries act as sink for defects: absorbing defects in front of them as they move and leaving relatively perfect material in their wake. In classical theories of grain boundary migration, the grain boundary velocity is proportional to the difference in energy density between the two sides of the boundary (i.e., the driving force) and the boundary mobility (that varies with grain boundary crystallography and material). The difference in energy between adjacent grains in ion beam assisted deposition (as described above) may drive the grain boundary towards the more defected (higher energy) grain. In order to determine whether this process actually occurs during IBAD of polycrystalline films, we performed simulations in which we damaged one end of a [111] oriented crystal with an ion beam, rotated the crystal and put its damaged surface in contact with an undamaged [110] oriented crystal (thereby creating a grain boundary), and monitored the position of the grain boundary as a function of time. The resultant structures are shown in 


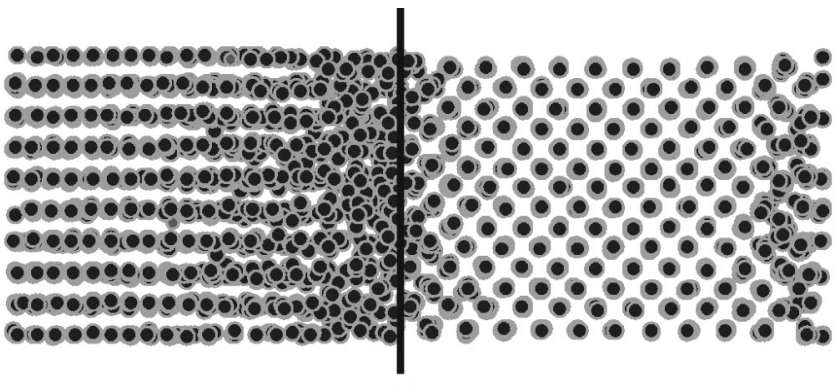

(a)

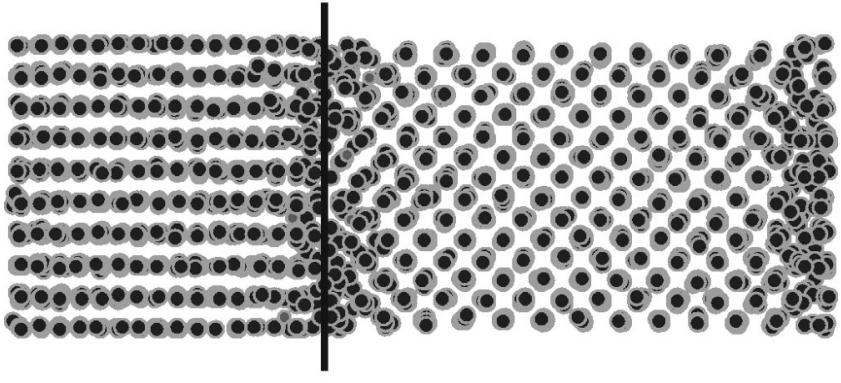

(b)

FIG. 12. Atomic structure of the bicrystal formed by joining the ion beam damaged end of a [111] crystal with an undamaged [110] crystal at (a) $t$ $=0.2 \tau$ and (b) $t=10 \tau$. The solid line indicates the position of the central grain boundary (another exists at the edge of the bicrystal due to the use of periodic boundary conditions in the $x$ direction). The grain boundary clearly migrates into the damaged [111] grain.

Fig. 12 at $t=0.2 \tau$ and $10 \tau$. The [111] grain is initially heavily damaged in a thin region near the grain boundary. The grain boundary clearly migrates into the damaged region of the [111] crystal and, hence, the [110] grain grows at the expense of the [111] grain. At the same time, the other grain boundary where the undamaged end of the [111] crystal meets the undamaged [110] crystal remains at very near its original position (the right edge of the periodic bicrystal in Fig. 12). This is a graphic demonstration of ion beam induced recrystallization. These results suggest that the pinching off of the [111] grain by the surrounding [110] grains by grain boundary migration in the bicrystal IBAD simulations shown in Fig. 2 is a result of ion beam induced recrystallization.

\section{CONCLUSIONS}

We performed molecular dynamics simulations of ion beam assisted deposition to determine the mechanisms of crystallographic texture selection during the IBAD of polycrystalline films. In these simulations, a face centered cubic bicrystal consisting of $\langle 111\rangle$ and $\langle 110\rangle$ oriented grains was grown while an ion beam impacted the growing film at normal incidence. As the film grew, the grain boundaries delimiting the $\langle 111\rangle$ grain moved towards each other, eventually entirely pinching off the $\langle 111\rangle$ grain. During growth, the film texture changed from equal densities of $\langle 111\rangle$ and $\langle 110\rangle$ to purely $\langle 110\rangle$. Examination of single crystals grown in the presence of an ion beam showed two important effects: both (1) ion beam induced atomic sputtering from the surface and (2) ion beam induced damage are significantly reduced when the ion beam is oriented along channeling directions of the crystals. The first observation suggests that grains with channeling directions aligned parallel to the ion beam grow more quickly than those where they are not aligned. This leads to grain-to-grain variations in the film thickness that increase in magnitude during growth. Variations in thickness result in a shadowing effect that further slows the growth of the less thick (nonaligned) grains - eventually leading to pinch-off of the less thick grains. The second observation suggests that the energies of the grains with channeling directions aligned parallel to the ion beam will be lower than that of the nonaligned grains. This difference in stored energy (in the form of crystal defects) was shown to lead to grain boundary migration - a process equivalent to primary recrystallization. Both of these effects can lead to changes in crystallographic texture during film growth and both were observed in the bicrystal simulations. It remains unclear as to which effect will be dominant. Finally, we note that these ion beam induced texture changes will compete with other mechanisms that control texture evolution in the absence of an ion beam; namely, surface energy anisotropy induced grain boundary migration (so called, secondary grain growth) ${ }^{36}$ and preferential nucleation.

\section{ACKNOWLEDGMENTS}

The authors gratefully acknowledge useful discussions with Professor Gary Was and Mr. Zhenqiang Ma of the University of Michigan. This work was supported by the Defense Advanced Research Projects Agency and the Air Force Office of Scientific Research under Grant No. F49620-95-10524.

${ }^{1}$ G. N. Van Wyk and H. J. Smith, Nucl. Instrum. Methods Phys. Res. A 170, 443 (1980).

${ }^{2}$ D. Dobrev, Thin Solid Films 92, 41 (1982).

${ }^{3}$ L. S. Yu, J. M. E. Harper, J. J. Cuomo, and D. A. Smith, Appl. Phys. Lett. 47, 932 (1985).

${ }^{4}$ L. S. Yu, J. M. E. Harper, J. J. Cuomo, and D. A. Smith, J. Vac. Sci. Technol. A 4, 443 (1986)

${ }^{5}$ W. Ensinger, Nucl. Instrum. Methods Phys. Res. B 106, 142 (1995).

${ }^{6}$ Y. Nagai, A. Tago, and T. Toshima, J. Vac. Sci. Technol. A 5, 61 (1987).

${ }^{7}$ E. Kay, F. Parmigiani, and W. Parrish, J. Vac. Sci. Technol. A 6, 3074 (1988).

${ }^{8}$ F. A. Smidt, Int. Mater. Rev. 35, 61 (1990).

${ }^{9}$ L. Tang and G. Thomas, J. Appl. Phys. 74, 5025 (1993).

${ }^{10}$ D. B. Knorr and D. P. Tracy, Appl. Phys. Lett. 59, 3241 (1991).

${ }^{11}$ D. B. Knorr and T.-M. Lu, Textures Microstruct. 13, 155 (1991).

${ }^{12}$ A. Van der Drift, Philips Res. Rep. 22, 267 (1967).

${ }^{13}$ M. B. Stearns, C. H. Lee, C.-H. Chang, and A. K. Petford-Long, in Metallic Multilayers and Epitaxy, edited by M. Hong, S. Wolf, and C. C. Gubser (TMS, Warrendale, PA, 1988), p. 55.

${ }^{14}$ R. W. Smith, J. Appl. Phys. 81, 1196 (1997).

${ }^{15}$ P. Sigmund, Phys. Rev. 184, 383 (1969).

${ }^{16}$ O. Karpenko, J. C. Bilello, and S. M. Yalisove, J. Appl. Phys. 82, 1397 (1997).

${ }^{17}$ F. Ying, R. W. Smith, and D. J. Srolovitz, Appl. Phys. Lett. 69, 3007 (1996).

${ }^{18}$ R. M. Bradley, J. M. E. Harper, and D. A. Smith, J. Appl. Phys. 60, 4160 (1986).

${ }^{19}$ R. M. Bradley, J. M. E. Harper, and D. A. Smith, J. Vac. Sci. Technol. A 5, 1792 (1987).

${ }^{20}$ T. J. Whetten, A. A. Armstead, T. A. Grzybowski, and A. L. Ruoff, J. Vac. Sci. Technol. A 2, 477 (1984).

${ }^{21}$ T. W. Snouse and L. C. Haughney, J. Appl. Phys. 37, 700 (1966). 
${ }^{22}$ H. E. Roosendaal, in Topics in Applied Physics, edited by R. Behrisch (Springer, Berlin, 1981), Vol. 47, Chap. 5.

${ }^{23}$ W. Möller, Nucl. Instrum. Methods Phys. Res. B 89, 322 (1994).

${ }^{24}$ K.-H. Müller, Phys. Rev. B 35, 7906 (1987).

${ }^{25}$ A. M. Mazzone, Thin Solid Films 216, 145 (1992).

${ }^{26}$ M. Posselt and K.-H. Heinig, Nucl. Instrum. Methods Phys. Res. B 102, 246 (1995).

${ }^{27}$ Z. Insepov and I. Yamada, Nucl. Instrum. Methods Phys. Res. B 99, 248 (1995).

${ }^{28}$ H. Gades and H. M. Urbassek, Phys. Rev. B 50, 11167 (1994).

${ }^{29}$ W. Möller, Thin Solid Films 228, 319 (1993).
${ }^{30}$ V. M. Konoplev, Nucl. Instrum. Methods Phys. Res. B 42, 229 (1989).

${ }^{31}$ W.-Z. Li, F.-Z. Cui, Y. Liao, and H.-D. Li, Vacuum 40, 289 (1990).

${ }^{32}$ W. Möller, D. Bouchier, O. Burat, and V. Stambouli, Surf. Coat. Technol. 45, 73 (1991).

${ }^{33}$ L. Dong, R. W. Smith, and D. J. Srolovitz, J. Appl. Phys. 80, 5682 (1996). ${ }^{34}$ G. Molière, Z. Naturforsch. A 2, 133 (1947).

${ }^{35} \mathrm{~W}$. Eckstein, Computer Simulation of Ion-Solid Interactions (Springer, Berlin, 1991).

${ }^{36}$ C. C. Wong, H. I. Smith, and C. V. Thompson, Appl. Phys. Lett. 48, 335 (1986). 